\title{
Exogenous retroelement integration in sperm and embryos affects preimplantation development
}

\author{
C Kitsou ${ }^{1}$, L Lazaros ${ }^{1}$, S Bellou², G Vartholomatos ${ }^{3}$, P Sakaloglou ${ }^{1}$, E Hatzi ${ }^{1}$, S Markoula ${ }^{4}$, \\ K Zikopoulos ${ }^{1}$, T Tzavaras ${ }^{5}$ and I Georgiou ${ }^{1}$ \\ 'Laboratory of Medical Genetics and Human Reproduction, Medical School, Ioannina University, loannina, \\ Greece, ${ }^{2}$ Foundation for Research \& Technology-Hellas Institute of Molecular Biology and Biotechnology, \\ Department of Biomedical Research, Ioannina, Greece, ${ }^{3}$ Hematology Laboratory, Unit of Molecular Biology, \\ Ioannina University Hospital, Ioannina, Greece, ${ }^{4}$ Department of Neurology, Medical School, loannina University, \\ Ioannina, Greece and ${ }^{5}$ Department of General Biology, Medical School, loannina University, loannina, Greece
}

Correspondence should be addressed to I Georgiou; Email: igeorgio@uoi.gr

\begin{abstract}
Retroelement transcripts are present in male and female gametes, where they are typically regulated by methylation, noncoding RNAs and transcription factors. Such transcripts are required for occurrence of retrotransposition events, while failure of retrotransposition control may exert negative effects on cellular function and proliferation. In order to investigate the occurrence of retrotransposition events in mouse epididymal spermatozoa and to address the impact of uncontrolled retroelement RNA expression in early preimplantation embryos, we performed in vitro fertilization experiments using spermatozoa preincubated with plasmid vectors containing the human retroelements LINE-1, HERVK-10 or the mouse retroelement VL30, tagged with an enhanced green fluorescence (EGFP) gene-based cassette. Retrotransposition events in mouse spermatozoa and embryos were detected using PCR, FACS analysis and confocal microscopy. Our findings show that: (i) sperm cell incorporates exogenous retroelements and favors retrotransposition events, (ii) the inhibition of spermatozoa reverse transcriptase can decrease the retrotransposition frequency in sperm cells, (iii) spermatozoa can transfer exogenous human or mouse retroelements to the oocyte during fertilization and (iv) retroelement RNA overexpression affects embryo morphology and impairs preimplantation development. These findings suggest that the integration of exogenous retroelements in the sperm genome, as well as their transfer into the mouse oocyte, could give rise to new retrotransposition events and genetic alterations in mouse spermatozoa and embryos.

Reproduction (2016) 152 185-193
\end{abstract}

\section{Introduction}

Retroelements are a major type of genetic mobile elements that occupy $43 \%$ of the human and $37 \%$ of the mouse genome (Lander et al. 2001, Waterston et al. 2002). They have played a crucial role in genome structure, function and evolution by relocating themselves from site to site in the genome through an RNA intermediate. The process of their mobilization or retrotransposition requires a transcription step, by a cellular RNA polymerase, followed by reverse transcription of the ensuing RNA intermediate into cDNA and its integration into a new genomic locus. Despite their high distribution in the mammalian genomes, only a minority of them is still retrotransposition-active, as they have lost their capacity to retrotranspose, due to defective mutations and truncations (Moran et al. 1996, Ostertag \& Kazazian 2001).
Based on structural and functional criteria, retroelements are classified by LTR and nonLTR retrotransposons as well as autonomous and nonautonomous retrotransposons. The human LTR retrotransposons include the endogenous retroviruses (ERVs), whereas the non-LTR retrotransposons include the major classes of LINEs and SINEs. Retroelements, encoding themselves reverse transcriptase and other proteins necessary for their mobilization, can relocate autonomously (Kazazian 2004). Active retroelements and heritable retrotranspositions have been documented in the germline and in embryos during the early stages of development (Kano et al. 2009, Rosser \& An 2012). LINE-1, SVA and HERVK-10 retroelements are transcriptionally expressed in human oocytes (Georgiou et al. 2009). SINEs and LINEs are also highly expressed during spermatogenesis (Miller 2000, Ergun et al. 2004, Krawetz et al. 2011). LINE-1, a family of 
non-LTR autonomous retroelements, mediates the retrotransposition of nonautonomous retroelements such as Alus and SVAs (Ostertag \& Kazazian 2001, Goodier \& Kazazian 2008), while HERVs constitute a major family of endogenous retroviruses.

Living spermatozoa of almost all species have been found to take up spontaneously exogenous DNA and internalize a part of it into their nucleus (Francolini et al. 1993). A small proportion of the internalized DNA is integrated at specific sites in sperm genome, probably at a nucleosomal subfraction of chromatin, highly enriched in under-methylated retrotransposon sequences, suggesting a common site for exogenous DNA integration and endogenous retrotransposition events (Zoraqi \& Spadafora 1997, Pittoggi et al. 2000). The uptake and the reverse transcription of exogenous RNA molecules to cDNA by mouse epididymal spermatozoa revealed a reverse transcriptase activity (Giordano et al. 2000). In addition, their incubation with a plasmid, containing a green fluorescence (EGFP) retrotransposition cassette interrupted by an intron with opposite orientation to the EGFP gene, has documented the presence of an active transcriptional machinery in spermatozoa, capable of mediating transcription, splicing and reverse transcription (Pittoggi et al. 2006).

Taking into account the occurrence of retrotransposition events during spermatogenesis, the capability of sperm cells to integrate exogenous DNA into their genome as well as the presence of an active machinery capable of mediating transcription, splicing and reverse transcription, we sought to clarify whether the internalization of exogenous retroelements into sperm genome could favor retrotransposition events as observed previously in human oocytes (Georgiou et al. 2009). In this study, we incubated mouse epididymal spermatozoa with plasmid vectors containing either the human LINE-1 or HERVK-10 or the mouse VL30 retroelement, tagged with an enhanced green fluorescence (EGFP) gene-based retrotransposition cassette and investigated the occurrence of de novo retrotransposition events in mouse spermatozoa. Also, we investigated the potential transfer of these retroelements into the mouse oocyte during fertilization, as well as their impact on the mouse preimplantation embryo development.

\section{Materials and methods}

\section{Animals}

Inbred FVB/N male and female mice were obtained from the Breeding Laboratory Animal Institute (Ioannina University Medical School, Greece). Animals were kept under a controlled $12 \mathrm{~h}$ light: $12 \mathrm{~h}$ darkness life cycle with a standard diet and free access to water. The General Directorate of Agricultural Economics and Veterinary Medicine of Epirus Region, Greece, approved the research protocols used.
Animals were treated in compliance with the European Union guidelines for the care and use of animals approved by our institution in accordance to principles of laboratory animal breeding and handling.

\section{Retrotransposition assay}

The presence of de novo retrotransposition events in mouse spermatozoa and embryos was studied using an indicator cassette of retrotransposition based on CMV promoter-driven EGFP expression (Ostertag et al. 2000). Specifically, in this cassette: the EGFP gene is transcriptionally controlled by a CMV immediate early promoter, but its expression is interrupted by a $\gamma$-globin intron cloned in opposite transcriptional orientation to that of EGFP. As exemplified for retrotransposon L1 (Fig. 1), the cassette is cloned into the $3^{\prime}$-UTR and in opposite transcription orientation to that of L1. Accordingly, the EGFP expression can only be detected when recombinant retrotransposon transcripts undergo splicing, reverse transcription and integration of the ensuing cDNA into the chromosomal DNA, mirroring a retrotransposition event. In the current study, we used the EGFP-tagged retrotransposons $\mathrm{pL} 1_{\mathrm{PR}}-\mathrm{EGFP}$ for human LINE-1 (Ostertag et al. 2000), pHERVK-10/ EGFP-INT (unpublished construct, contributed by Prof. T Tzavaras) for human HERVK-10 and pNVL-3*/ EGFP-INT for mouse VL30 (Noutsopoulos et al. 2006)

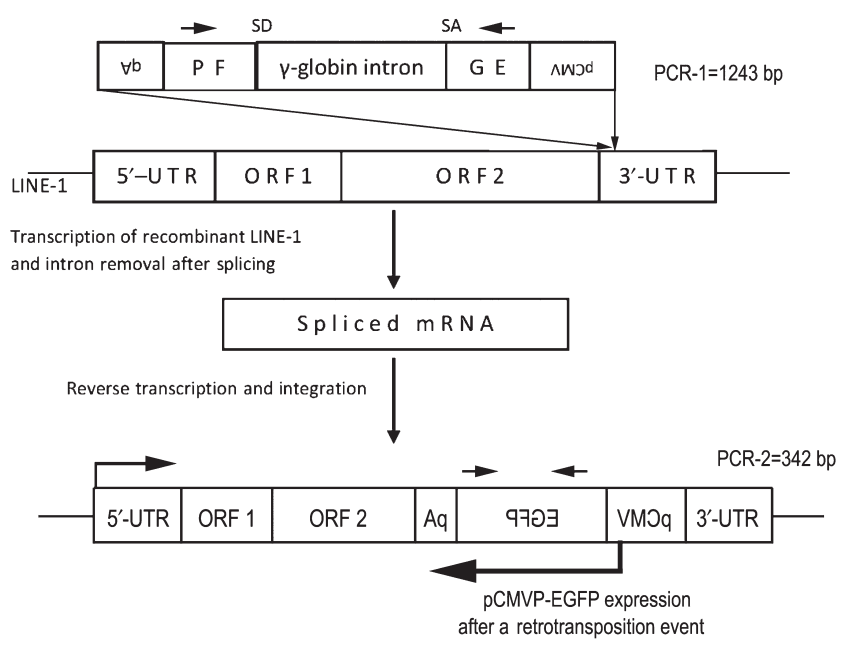

Figure 1 Schematic presentation of a human LINE-1 tagged with an indicator EGFP retrotransposition cassette. The EGFP retrotransposition cassette is cloned in the $3^{\prime}$-UTR of LINE- 1 and in antisense orientation. The expression of EGFP gene is interrupted by a $\gamma$-globin intron and is only expressed after the steps of transcription, splicing, reverse transcription and integration of the ensuing CDNA into the chromosomal DNA, mirroring a retrotransposition event. Small opposite arrows above EGFP gene show PCR primers.

PCR $-1=1243 \mathrm{bp}$ and PCR-2 = $342 \mathrm{bp}$ denote PCR products before and after retrotransposition respectively. Long arrows at the $5^{\prime}$ UTR and $\mathrm{PCMV}$ show transcription orientations from retrotransposon LINE-1 and EGFP gene respectively. 
respectively. In addition, a mutant, nonfunctional LINE-1 retrotransposition cassette was used as negative control, containing the EGFP gene without the antisense $\gamma$-globin intron (Ostertag et al. 2000, Noutsopoulos et al. 2006), while the pEGFP-N1 (Clontech) was used as a positive control for EGFP expression.

\section{Sperm preparation}

Male mice 10-12 weeks old with proven fertility that had not been mated for 3-7 days were killed by cervical dislocation and sperm was collected from cauda epididymis in culture medium (Sydney IVF Sperm Medium, COOK, Limerick, Ireland) under mineral oil (Sydney IVF Culture Oil, COOK, Limerick, Ireland). Motile spermatozoa were selected using the swim up method and counted in a Neubauer chamber. They were used for IVF following fluorescence-activated cell scanning (FACS) and confocal microscopic analysis.

For IVF experiments, an amount of sperm was left to be capacitated in fertilization medium (Sydney IVF Fertilization Medium, COOK, Limerick, Ireland) for $1.5 \mathrm{~h}\left(37^{\circ} \mathrm{C}, 5 \% \mathrm{CO}_{2}\right)$ under mineral oil. The last $30 \mathrm{~min}$ of the capacitation, sperm samples were incubated with five different plasmid vectors (at concentration of $50 \mathrm{ng} / 10^{6}$ sperm cells) containing respectively the human LINE-1, human HERVK-10, mouse VL30, pEGFP-N1 and mutant nonfunctional LINE-1 and then added to the fertilization plates (200 sperm cells/ $\mu \mathrm{L}$ ).

For FACS analysis, an amount of motile spermatozoa was incubated with plasmid vectors containing the human retrotransposons LINE-1 or HERVK-10 or the mouse retrotransposon VL30 (at concentrations of 50, 100, 200 and $400 \mathrm{ng} / 10^{6}$ sperm cells), the pEGFP-N1 and the mutant nonfunctional LINE-1 for 5, 12 and $24 \mathrm{~h}\left(37^{\circ} \mathrm{C}, 5 \% \mathrm{CO}_{2}\right)$. In inhibitory experiments, the nucleoside analog reverse transcriptase inhibitor Lamivudine (Sigma Aldrich, Life Science Chemilab A.E., Athens, Greece) was prepared in DMSO (Sigma Aldrich, Life Science Chemilab A.E., Athens, Greece) at concentrations of 15, 25, 50, 200, 400 and $800 \mu \mathrm{M}$ and added to the sperm suspension at a final volume of $0.5 \% \mathrm{v} / \mathrm{v}, 2 \mathrm{~h}$ before the sperm-plasmid vectors incubation (at concentrations of $50 \mathrm{ng} / 10^{6}$ sperm cells). The same volume (DMSO $0.5 \% \mathrm{v} / \mathrm{v}$ ) with absence of Lamivudine was also added to the control samples. For confocal microscopy, sperm samples were mounted on a microscope slide and observed for EGFP fluorescence.

\section{Oocyte collection}

Superovulation in 6-8-weeks-old female mice was induced with an intraperitoneal injection of $8 \mathrm{IU}$ follicle-stimulating hormone (rec-FSH, Gonal-F, Merk Serono, London, UK) followed by an intraperitoneal injection of $5 \mathrm{IU}$ human chorionic gonadotropin (hCG, Pregnyl, N.V. Organon, Oss, The Netherlands) after $48 \mathrm{~h}$. The mice were killed by cervical dislocation 13-h post-hCG treatment and oocytes were collected from the oviducts in culture medium (Sydney IVF Follicle Flush Buffer \& Fertilization Medium, COOK, Limerick, Ireland) under mineral oil.

\section{IVF procedure}

Mouse oocytes were incubated with sperm in fertilization plates for $5 \mathrm{~h}$, and washed in culture medium (Sydney IVF Fertilization Medium, COOK, Limerick, Ireland) to remove sperm excess. The fertilization outcome was monitored by the presence of the 2 nd polar body and the two pronuclei formed. Zygotes were cultured in cleavage and blastocyst medium (Sydney IVF Cleavage Medium \& Sydney IVF Blastocyst Medium, COOK, Limerick, Ireland) until the stage of blastocyst $\left(37^{\circ} \mathrm{C}, 5 \% \mathrm{CO}_{2}\right)$. Embryos preimplantation development was evaluated every day.

\section{FACS analysis}

Sperm cells were washed twice and resuspended in PBS. A total number of 10,000 sperm cells per sample were analyzed with FACS for the determination of retrotransposition frequency. Nonincubated sperm cells were used as controls to evaluate background fluorescence setting intensity thresholds. Additionally, mutant, nonfunctional LINE-1-incubated sperm cells were used as negative controls while pEGFP-N1incubated sperm cells as positive controls. Sample values of up to $99.6 \%$ in the FACS curve were considered as negative and $0.4 \%$ as false positive. Sample values above the $0.4 \%$ threshold were considered as positive (Noutsopoulos et al. 2006). Data analysis was performed by the FACSCalibur cytometer using the CellQuest program.

\section{Confocal microscopy}

Sperm samples and living mouse preimplantation embryos were viewed using Leica TCS SP5 scanning laser confocal microscope, equipped with an argon laser (for excitation at $488 \mathrm{~nm}$ ), solid-state 561 laser line and helium-neon laser (for excitation at $633 \mathrm{~nm}$ ). Objective lens used was Leica X10 and images were obtained using the LASAF Software.

\section{PCR analysis}

After FACS analysis, mouse sperm samples were analyzed by PCR in order to confirm the presence or absence of retrotransposition events. Specifically, DNA was extracted from treated sperm using the QIAmp DNA Mini Kit (Qiagen) with minor modifications. In brief, up to $5 \times 10^{6}$ spermatozoa were mixed with $20 \mu \mathrm{L}$ of proteinase Kand $100-200 \mu \mathrm{L}$ of buffer $X(20 \mathrm{mM}$ Tris-HCl pH 8.0, $20 \mathrm{mM}$ EDTA, $200 \mathrm{mM}$ $\mathrm{NaCl}, 80 \mathrm{mM}$ DTT, $4 \%$ SDS). After incubation at $56^{\circ} \mathrm{C}$ for $1 \mathrm{~h}, 200 \mu \mathrm{L}$ of AL buffer and $200 \mu \mathrm{L}$ of absolute ethanol were added in each sample and the subsequent steps of the 'blood and body fluid protocol' QIAmp DNA Mini Kit (Qiagen) were followed. The final elution of DNA was done in $80 \mu \mathrm{L}$ of distilled water. The PCR analysis was performed according to a protocol described previously (Noutsopoulos et al. 2006). Briefly, two EGFP oligonucleotides were used: GFP968F 5'-GCACCATCTTCTTCAAGGACGAC-3' and GFP1013R 5'-TCTTTGCTCAGGGCGGACTG-3' and the amplification was performed in a $50 \mu \mathrm{L}$ reaction containing $10 \mu \mathrm{L}$ of the spermatozoa-extracted DNA. The thermal cycling was as 
follows: denaturation at $94^{\circ} \mathrm{C}$ for $15 \mathrm{~min}, 35$ cycles at $94^{\circ} \mathrm{C}$ for $30 \mathrm{~s}, 59^{\circ} \mathrm{C}$ for $30 \mathrm{~s}$ and $72^{\circ} \mathrm{C}$ for $1.5 \mathrm{~min}$, with a final extension at $72^{\circ} \mathrm{C}$ for $10 \mathrm{~min}$.

After confocal screening, embryos were also analyzed by PCR so as to confirm the presence or absence of retrotransposition events. Each embryo was placed into a $0.2 \mathrm{~mL}$ Eppendorf tube containing $10 \mu \mathrm{L}$ double-distilled sterile water and PCR-grade proteinase K (Qiagen) to a final concentration of $50 \mu \mathrm{g} / \mathrm{mL}$. Embryo lysis was performed with proteinase $\mathrm{K}$ by incubation at $37^{\circ} \mathrm{C}$ for $1 \mathrm{~h}$ followed by $56^{\circ} \mathrm{C}$ for $10 \mathrm{~min}$ and proteinase $\mathrm{K}$ inactivation by heating to $95^{\circ} \mathrm{C}$ for $10 \mathrm{~min}$. The subsequent PCR and nested PCR analysis (35 and 25 cycles respectively) were performed according to the above protocol with minor modifications. PCR products were separated by $2 \%$ agarose gel electrophoresis and visualized by exposure to ultraviolet light after ethidium bromide staining.

For the estimation of the copy number of integrated retrotransposon sequences per genome, we compared the density of EGFP and $\beta$-actin, a single copy endogenous gene, PCR bands in embryos positive for retrotransposition events. The lysis of embryos was achieved through activation of proteinase $\mathrm{K}$ by incubation at $37^{\circ} \mathrm{C}$ for $1 \mathrm{~h}$ followed by $56^{\circ} \mathrm{C}$ for $30 \mathrm{~min}$ and proteinase $\mathrm{K}$ inactivation by heating to $95^{\circ} \mathrm{C}$ for $20 \mathrm{~min}$. Lysates were used for EGFP and $\beta$-actin PCR in separate reactions. The PCR cycling of both analyses was as follows: denaturation at $94^{\circ} \mathrm{C}$ for $15 \mathrm{~min}, 40$ cycles of $94^{\circ} \mathrm{C}$ for $30 \mathrm{~s}, 59^{\circ} \mathrm{C}$ for $30 \mathrm{~s}$ and $72^{\circ} \mathrm{C}$ for $1.5 \mathrm{~min}$, with a final extension at $72^{\circ} \mathrm{C}$ for $10 \mathrm{~min}$. As concerns the $\beta$-actin PCR analysis, the following primer pair was used: F: 5'-GTGACGTTGACATCCGTAAAG-3' and R: 5'-GCCGGACTCATCGTACTC-3'. PCR products were separated by $2 \%$ agarose gel electrophoresis and visualized by exposure to ultraviolet light after ethidium bromide staining. Densitometry band analysis was performed by ImageJ software.

\section{Statistical analysis}

FACS analysis was evaluated by the Kolmogorov-Smirnov test. Measurements with $P \leq 0.001, \mathrm{D} / \mathrm{s}(\mathrm{n}) \geq 12$ and $\mathrm{D} \geq 0.12$ were set as statistically significant. Statistical analysis for the mean retrotransposition frequency in sperm samples was performed using the ANOVA and the t-test, by the SPSS (version 20).

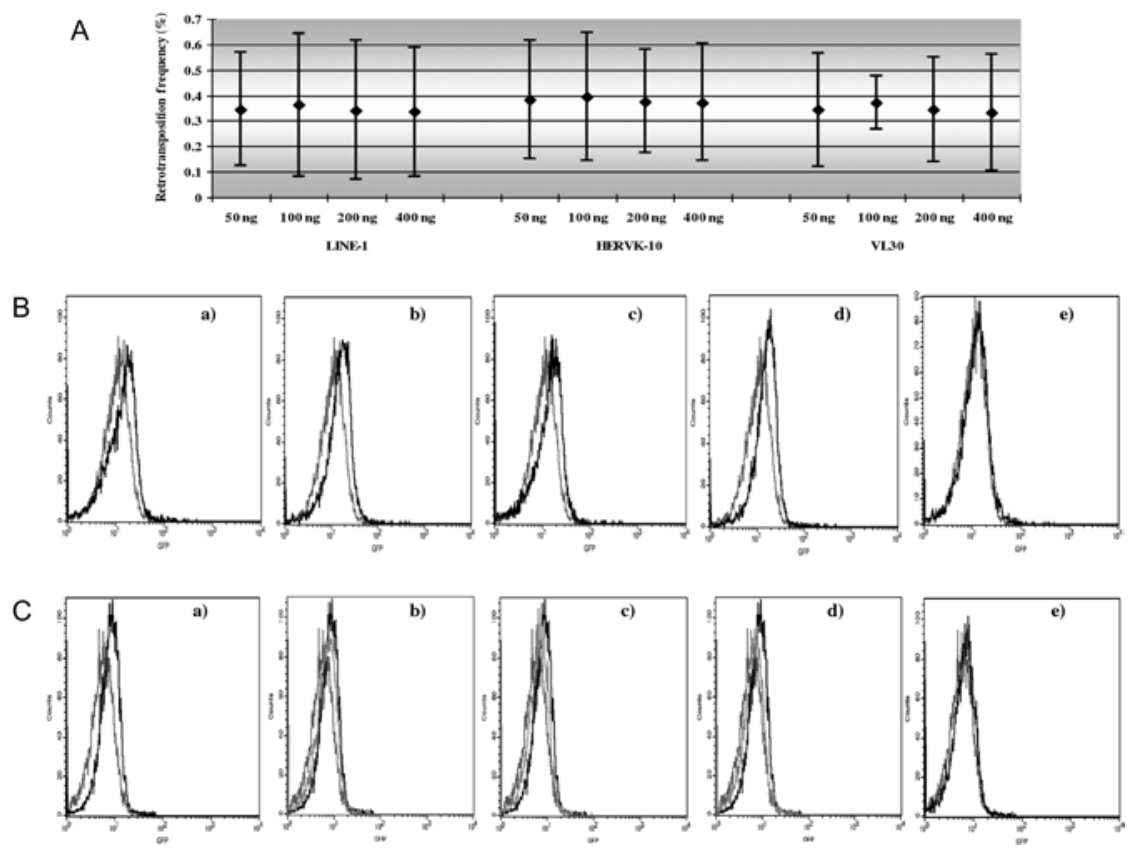

Figure 2 (A) Retrotransposition frequency of human retroelements LINE-1, HERVK-10 and mouse retroelements VL30 in mouse epididymal spermatozoa after a 5-h incubation with 50, 100, 200 and $400 \mathrm{ng} / 10^{6}$ sperm cells as found by FACS analysis. (B) FACS analysis of HERVK-10 retrotransposition frequency in mouse epididymal spermatozoa. Graphs show analyses of a representative experiment of sperm samples without exogenous plasmid incubation (gray curve) and after a 5-h incubation with (a) 50, (b) 100, (c) 200 and (d) 400 ng/10 sperm cells HERVK-10 plasmid vector (black curve) and (e) $50 \mathrm{ng} / 10^{6}$ sperm cells mutant, nonfunctional LINE-1 plasmid vector (black curve). The sperm sample incubated with HERVK-10 showed a slight increase in retrotransposition frequency from $50 \mathrm{ng}(0.65 \%)$ to $100 \mathrm{ng} / 10^{6}$ sperm cells $(0.8 \%)$, which was diminished at $200(0.58 \%)$ and $400 \mathrm{ng} / 10^{6}$ sperm cells $(0.59)$. No retrotransposition events were observed after incubation with the mutant LINE-1 retrotransposition cassette. (C) FACS analysis of the inhibition of LINE-1 retrotransposition in mouse epididymal spermatozoa. Graphs show analyses of a representative experiment of sperm samples not incubated with exogenous plasmid (gray curve) and of sperm samples (a) after a 5-h incubation with $50 \mathrm{ng} / 10^{6}$ sperm cells of LINE-1 plasmid vector (black curve), after a $2 \mathrm{~h}$ pretreatment with increasing concentrations (b) $15 \mu \mathrm{M}$, (c) $25 \mu \mathrm{M}$ or (d) $50 \mu \mathrm{M}$ of reverse transcriptase inhibitor Lamivudine, followed by a 5 -h incubation with $50 \mathrm{ng} / 10^{6}$ sperm cells of LINE-1 plasmid vector (red curve). Graph (e) is representative of sperm samples after a 5 -h incubation with $50 \mathrm{ng} / 10^{6} \mathrm{sperm}$ cells of mutant, nonfunctional LINE-1 plasmid vector (black curve). The LINE-1 retrotransposition frequency (a) $0.25 \%$ was decreased (black curve) to (b) $0.14 \%$ at $15 \mu \mathrm{M}$ (red curve) to (c) $0.08 \%$ at $25 \mu \mathrm{M}$ (red curve) and (d) was totally inhibited to $0 \%$ at $50 \mu \mathrm{M}$ of Lamivudine (red curve). (e) No retrotransposition events observed after incubation with the mutant, nonfunctional LINE-1 retrotransposition cassette (black curve). 


\section{Results}

\section{Retrotransposition events in mouse epididymal spermatozoa}

To investigate whether retrotransposition events occur in mouse spermatozoa, we incubated spermatozoa with plasmid vectors containing the human retroelements LINE-1 or HERVK-10 or the mouse retroelement VL30 tagged with an EGFP cassette. The cassette is constructed to allow the expression of EGFP only when de novo retrotransposition events have taken place.

For the determination of the retrotransposition frequency, mouse epididymal spermatozoa were incubated with the exogenous retroelements LINE-1, HERVK-10 and VL30 in escalating concentrations (50, 100, 200 and $400 \mathrm{ng} / 10^{6}$ sperm cells) for 5, 12 and $24 \mathrm{~h}$. From the 53 studied sperm samples, 28 $(52.8 \%)$ were found positive for retrotransposition. The retrotransposition frequency was not statistically different between retroelements (Fig. 2A), was not dose (Fig. 2B) and time dependent, which led us to implement a 5-h incubation period in our experiments. Specifically, the mean \pm S.D. retrotransposition frequency of sperm samples incubated with HERVK-10 was $0.38 \pm 0.23 \%$ at $50 \mathrm{ng}, 0.39 \pm 0.25 \%$ at $100 \mathrm{ng}, 0.37 \pm 0.22 \%$ at $200 \mathrm{ng}$ and $0.37 \pm 0.22 \%$ at $400 \mathrm{ng}$ (corresponding to 38,39 , 37 and 37 sperm cells in 10.000 respectively). For sperm samples incubated with LINE-1, the retrotransposition frequency was $0.34 \pm 0.22 \%$ at $50 \mathrm{ng}, 0.36 \pm 0.28 \%$ at $100 \mathrm{ng}, 0.34 \pm 0.27 \%$ at $200 \mathrm{ng}$ and $0.33 \pm 0.25 \%$ at $400 \mathrm{ng}$ (corresponding to 34, 36, 34 and 33 sperm cells in 10.000 respectively) and for sperm samples incubated with VL30 was $0.34 \pm 0.22 \%$ at $50 \mathrm{ng}, 0.37 \pm 0.1 \%$ at $100 \mathrm{ng}, 0.34 \pm 0.2 \%$ at $200 \mathrm{ng}$ and $0.33 \pm 0.22 \%$ at $400 \mathrm{ng}$ (corresponding to 34, 37, 34 and 33 sperm cells in 10.000 respectively).

In order to inhibit reverse transcription of the intermediate mRNA, expressed from recombinant retrotransposons into CDNA, and consequently decrease de novo retrotransposition events, we used the nucleoside analog reverse transcriptase inhibitor, Lamivudine. Sperm samples were preincubated for $2 \mathrm{~h}$ with 15, 25, 50, 200, 400 and $800 \mu \mathrm{M}$ Lamivudine before incubation of sperm with the plasmid vectors (containing LINE-1, HERV-K10, VL30 at concentration of $50 \mathrm{ng} / 10^{6}$ sperm cells). FACS analysis showed a significant reduction in the retrotransposition frequency at the lower Lamivudine concentrations $(15-50 \mu \mathrm{M})$, particularly at $50 \mu \mathrm{M}$, (Fig. 2C), while at higher Lamivudine concentrations $(200-800 \mu \mathrm{M})$, the inhibitory action was less effective. Specifically, for LINE-1, the retrotransposition frequency $(0.34 \pm 0.22 \%)$ was reduced to $0.19 \pm 0.12 \%(15 \mu \mathrm{M}), 0.12 \pm 0.07 \%$ $(25 \mu \mathrm{M}), 0.02 \pm 0.01 \%(50 \mu \mathrm{M}), 0.28 \pm 0.18 \%(200 \mu \mathrm{M})$, $0.29 \pm 0.19 \%(400 \mu \mathrm{M})$ and $0.31 \pm 0.2 \%(800 \mu \mathrm{M})$ (data for HERVK-10 and VL30 are not shown).

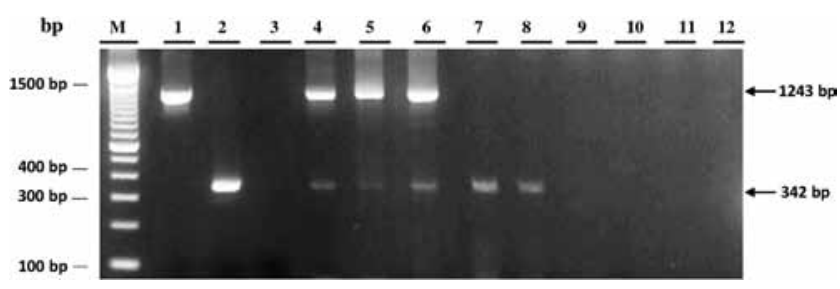

Figure 3 PCR analysis of single mouse embryos and mouse epididymal spermatozoa. Oocytes were fertilized by spermatozoa preincubated with the plasmid vector containing the human retrotransposons LINE-1 (50 ng/1 $10^{6}$ sperm cells) for the detection of retrotransposition events. Spermatozoa were incubated with $50 \mathrm{ng} / 10^{6}$ sperm cells of LINE-1, VL30 and HERVK-10. The $1243 \mathrm{bp}$ band represents the plasmid vectors containing the EGFP with the $\gamma$-globin intron and the $342 \mathrm{bp}$ band represents the diagnostic for retrotransposition events band. 1. Reaction with the pL1RP-EGFP control plasmid containing EGPF with the $\gamma$-globin (1243 bp), 2. reaction with the pEGFP-N1 control plasmid containing EGFP without the $\gamma$-globin (342bp), 3. blank sample, 4. HERVK-10 retrotransposition-positive sperm samples ( $50 \mathrm{ng} / 10^{6} \mathrm{sperm}$ cells), 5. LINE-1 retrotransposition-positive sperm samples $\left(50 \mathrm{ng} / 10^{6} \mathrm{sperm}\right.$ cells), 6. VL30 retrotransposition-positive sperm samples $\left(50 \mathrm{ng} / 10^{6}\right.$ sperm cells), 7. LINE-1 retrotransposition-positive embryo (blastocyst stage), 8. LINE-1 retrotransposition-positive embryo (morula stage), 9 and 10. LINE-1 retrotransposition-negative embryos, 11 and 12. control embryos.

To further confirm the presence or absence of retrotransposition events, sperm samples were subsequently analyzed by PCR. In the absence of retrotransposition, only a $1243 \mathrm{bp}$ PCR product was detected, which corresponds to the EGFP cassette containing the $\gamma$-globin intron. In contrast, sperm samples with active retrotransposition events, apart from
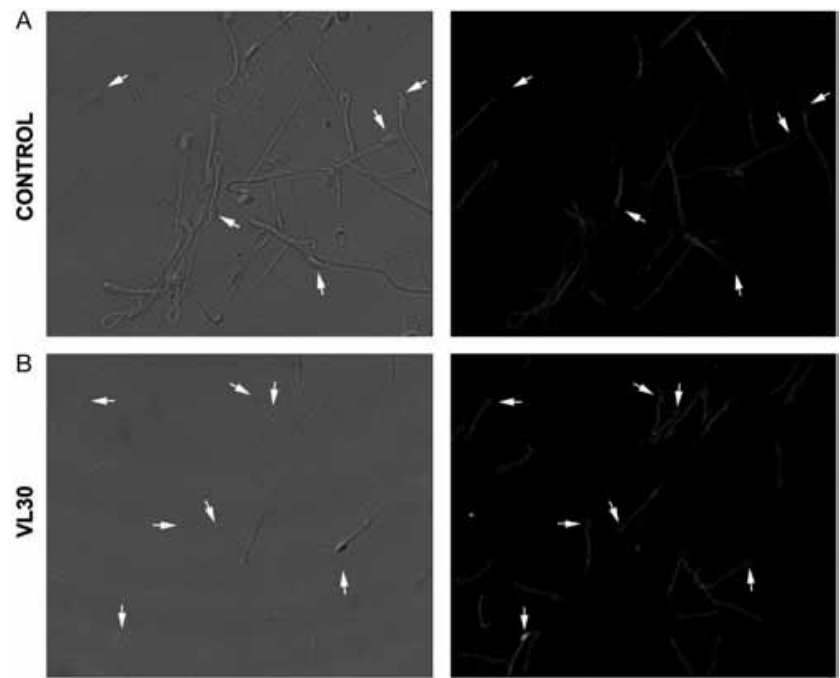

Figure 4 Confocal microscopy analysis of (A) control spermatozoa and (B) VL30-incubated spermatozoa. VL30-incubated spermatozoa positive for retrotransposition events present EGFP signal in their heads. 

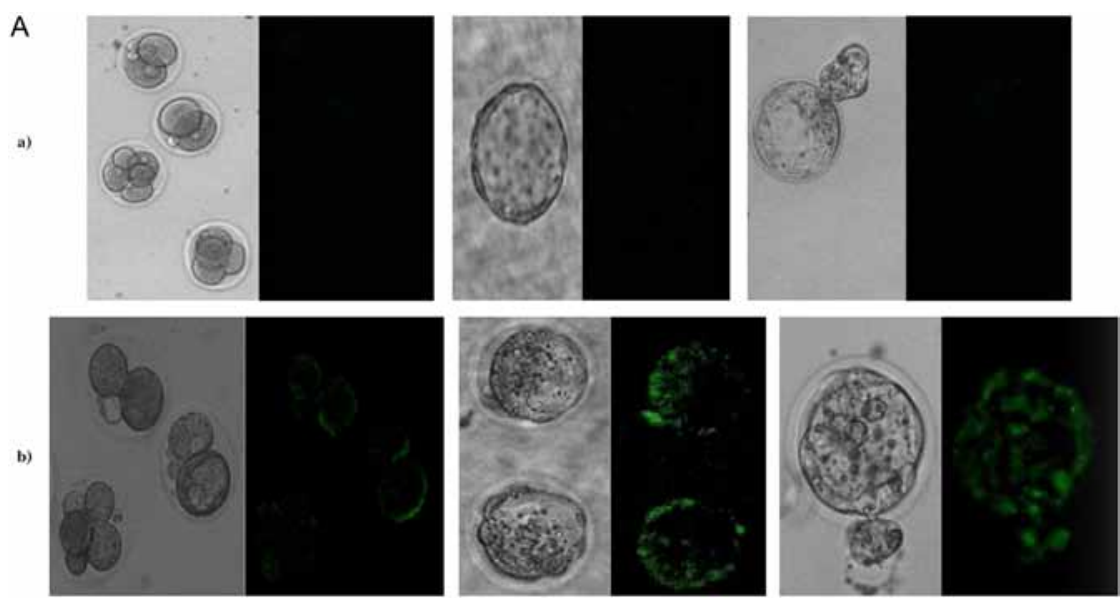

B

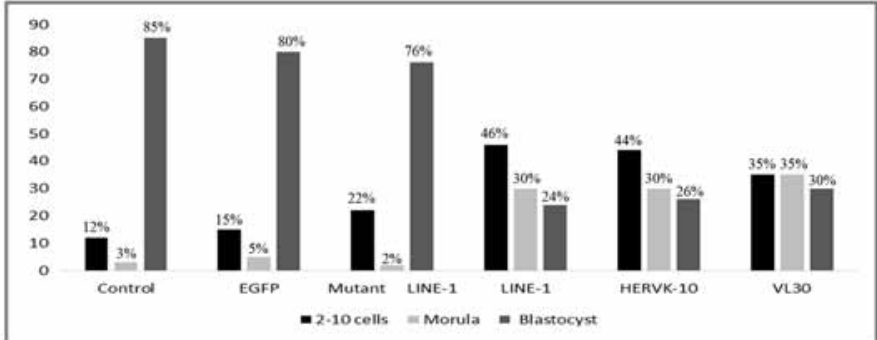

Figure 5 (A) Confocal microscopy analysis of (a) control embryos and (b) LINE-1 retrotransposition-positive embryos. (B) Preimplantation developmental stage at which retrotransposition event-positive embryos were arrested. the 1243 bp band, had also the 342 bp retrotranspositionspecific band (Fig. 3).

Sperm samples were also screened for EGFP expression by confocal microscopy. An EGFP fluorescent signal was observed in the heads of spermatozoa positive for retrotransposition events (Fig. 4).

Our results show that sperm cells can internalize constructs of cloned retroelements and favor retrotransposition events, the frequency of which can be hindered by reverse transcriptase inhibition.

The transfer of exogenous retroelements to mouse embryos through spermatozoa impairs embryo preimplantation development.

In order to explore the effect of retroelement DNA transfer to mouse oocytes during fertilization and to address the impact of retroelement expression in mouse early preimplantation development, we conducted IVF in mice using spermatozoa preincubated for $30 \mathrm{~min}$ with the plasmid vector containing the human retrotransposons LINE-1 or HERVK-10 or the mouse VL30 or the pEGFP-N1 and the mutant LINE-1 (50 ng $10^{6}$ sperm cells). The control group included 140 embryos, the LINE-1 group 114 embryos, the HERVK10 group 73 embryos, the VL30 group 65 embryos, the pEGFP-N1 group 40 embryos and the mutant LINE-1 group 45 embryos.

The presence of de novo retrotransposition events in embryos was evaluated through EGPF expression by confocal microscopy (Fig. 5A) and confirmed by PCR analysis. The existence of a $342 \mathrm{bp}$ band in PCR products was indicative of a functional EGFP protein, after $\gamma$-globin intron removal, and consequently due to occurrence of retrotransposition events (Fig. 3). The above analysis showed that $29 \%$ of the LINE-1 group of embryos, $37 \%$ of the HERVK-10 group of embryos and the $30 \%$ of the VL30 group of embryos were positive for retrotransposition events. The copy number of the integrated retrotransposon sequences per genome was estimated by comparison of the 342 bp EGFP band with the $349 \mathrm{bp} \beta$-actin band using the densitometric analysis

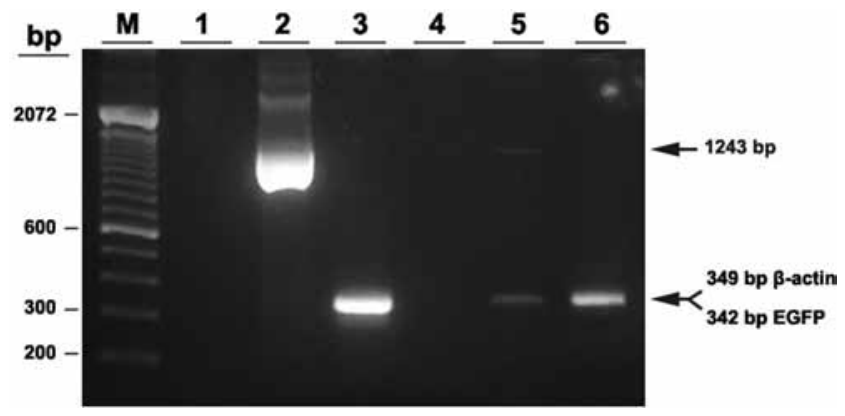

Figure 6 PCR analysis for the estimation of integrated retrotransposons sequences copies ratio in mouse embryos. Mouse oocytes were fertilized by spermatozoa preincubated with the plasmid vector containing the human retrotransposons LINE-1 ( $50 \mathrm{ng} / 10^{6}$ sperm cells). 1. Blank sample, 2. reaction with the pL1RP-EGFP control plasmid containing EGPF with the $\gamma$-globin (1243 bp), 3. reaction with the pEGFP-N1 control plasmid containing EGFP without the $\gamma$-globin (342 bp), 4. control embryo at blastocyst stage. 5. EGFP PCR product of a positive for retrotransposition embryo at the blastocyst stage, 6 . b-actin PCR product of a positive for retrotransposition embryo at the blastocyst stage. 
ImageJ. The relative density of bands was estimated in a ratio of $\sim 1: 4$, indicating that the integrated retrotransposon sequence copies are present in significantly less than one copy of active retrotransposon per genome (Fig. 6).

The in vitro preimplantation development of mouse embryos was scored daily until they reached the blastocyst stage. Embryos positive for retrotransposition were characterized by accelerated asymmetrical cell division, multiple cellular fragments, cleavage arrest and degeneration (Fig. 5A). The majority of retrotranspositionpositive embryos were arrested before blastocyst expansion and degenerated within the zona pellucida. Most of the retrotransposition-positive blastocysts had abnormal morphology and collapsed before hatching. The developmental stage at which retrotranspositionpositive embryos were arrested is represented in Fig. 5B. In brief, $85 \%$ of the control embryos reached the blastocyst stage, while only $24 \%$ of LINE-1-positive embryos, $26 \%$ of HERVK-10-positive embryos and $30 \%$ of VL30-positive embryos formed a blastocyst. Finally, $80 \%$ of pEGFP-N 1 embryos and $76 \%$ of mutant LINE-1 embryos reached the blastocyst stage indicating that the presence of the exogenous plasmid vector itself does not gravely affect preimplantation embryo development, as do the cloned active retroelements. Although a slight decrease in the numbers of blastocyst of mutant Line-1 group was observed, no statistically significant difference was found $(P>0.05)$, suggesting that in absence of any retrotransposition event, embryo development is not affected.

We conclude that the presence of active retroelements, expressed or transferred by spermatozoa that carry on their expression in the zygote, may impair mouse embryos at the later stages of preimplantation development.

\section{Discussion}

Retrotransposition events are more frequent in germ cells and in embryos at early developmental stages compared with somatic cells (Muotri et al. 2005, Kano et al. 2009). Retrotransposon transcripts have been documented in male and female gametes (Miller 2000, Georgiou et al. 2009, Krawetz et al. 2011), where their expression is typically under strict cellular control ensuring the normal cell genome function. DNA methylation constitutes the main regulator of endogenous retroelements expression, which is expected to be suppressed in hypermethylated nuclei and overexpressed in hypomethylated nuclei (Yu et al. 2001). In the current study, we investigated the occurrence of retrotransposition events in highly methylated mouse epididymal spermatozoa induced by cloned human and mouse retroelements and the consequences of cloned retrotransposon overexpression in mouse embryo preimplantation development. Taking into account the capability of mammalian spermatozoa to bind exogenous DNA or RNA molecules and internalize them into their nucleus (Francolini et al. 1993), we incubated spermatozoa with plasmid vectors containing active human LINE-1, HERVK-10 and mouse VL30 retroelements tagged with an EGFP retrotransposition cassette.

Mature spermatozoa, due to their highly compact DNA structure and their restricted cytoplasm, are thought to be transcriptionally and translationally inactive. Although various RNA types are transcribed during spermatogenesis and accumulate in mature spermatozoa, there is no evidence of newly synthesized RNA in the sperm cell (Grunewald et al. 2005, Krawetz et al. 2011). Furthermore, although transcriptional and translational activities in bovine sperm mitochondria (Premkumar \& Bhargava 1972) as well as mitochondrial-type ribosome translation during sperm capacitation (Gur \& Breitbart 2006) have been documented, nuclear activity has not yet been evidenced. However, the activation of an endogenous endonuclease in response to exogenous DNA in a dose-dependent manner, as well as the subsequent degradation of the internalized DNA as well as of the sperm cell DNA, leading to cell death through a metabolic pathway similar to apoptosis, supports the existence of sperm nuclear and cytoplasmic activity (Maione et al. 1997). Additionally, recent studies on the molecular mechanism of sperm-mediated gene transfer, indicated the presence of an active reverse transcriptase and also the presence of a transcriptional machinery, capable of catalyzing transcription, splicing and reverse transcription of an exogenous EGFP cassette into cDNA sequences (Pittoggi et al. 2006). Based on the study of Pittoggi and coworkers (2006), we used the EGFP retrotransposition cassette, additionally tagged with the human retroelements LINE-1, HEVK-10 and the mouse VL30, and we showed that spermatozoa favor retrotransposition events, through transcription, splicing, reverse transcription and integration of the recombinant retroelements into genomic loci. The integration sites possibly reside in the hypomethylated chromatin portion of the mouse sperm genome (Pittoggi et al. 2000). Furthermore, we verified the presence of retrotransposition events, by suppressing the activity of reverse trancriptase enzyme with Lamivudine. Indeed, the insertion rate of the exogenous retroelements was decreased, as monitored by FACS analysis. We hypothesize that sperm retrotransposition processes employ a transcriptional and translational machinery, possibly composed by factors produced in various stages of sperm growth and differentiation and stimulated by exogenous DNA of retrotransposon origin or by the sperm-mediated gene transfer mechanism itself.

Retrotransposition events were also confirmed in embryos indicating that spermatozoa convey the exogenous human or mouse retroelements to oocytes during in vitro fertilization. The exogenous DNA was 
possibly integrated into sperm genome and consequently transferred by the sperm and integrated in the genome of retrotransposition-positive embryos after IVF. The presence of excessive human or mouse retroelements in mouse fertilized oocytes by retrotranspositionpositive sperm impaired embryo preimplantation development. More specifically, the retroelements accelerated asymmetrical cell division, multiple cellular fragments, cleavage arrest at early developmental stages and embryo degeneration, while the majority of retrotransposition-positive blastocysts were characterized by impaired quality and collapse before hatching. In contrast, the absence of retrotransposition events in embryos obtained after oocyte fertilization with spermatozoa incubated with the pEGFP-N1 or the mutant, nonfunctional LINE-1 construct indicates that exogenous control plasmid vectors do not impair the in vitro mouse embryo preimplantation development.

The epigenetic reprogramming during the early embryo developmental stages may cause dynamic changes to the methylation patterns of the embryonic genome creating a favorable environment for retrotransposition events, which are potentially necessary for embryogenesis (van den Hurk et al. 2007). Under normal conditions, the expression of the retrotrasposable elements is controlled by RNA-interference mechanisms (Krawetz et al. 2011) and transcription factors (Becker et al. 1993, Kurose et al. 1995, Watanabe et al. 2006), whereas DNA repair mechanisms identify and repair double-strand DNA damages. However, the induction of retrotransposition events by exogenous human or mouse retroelements, which was accomplished in the current study, may have led to dysregulation of these control mechanisms resulting in abnormal embryo preimplantation development. A recent study has reported that failure of human retroelement control may negatively affect cellular proliferation, genomic stability and embryo development (Dimitriadou et al. 2013). Furthermore, many retrotransposition-induced mutagenesis cases and retrotransposition-derived disorders are presumably events that escape the retrotransposition control mechanisms (Kazazian 2004). Consequently, the incorporation of active exogenous retrotransposons may give rise to new, probably uncontrolled retrotrasposition events at the preimplantation stages. Indeed, the introduction of exogenous retroelements in somatic cells has been shown to affect their phenotype, differentiation and propagation (Tzavaras et al. 1998). The above findings, suggest that the increased rates of abnormal embryos found in the current study are possibly due to de novo retrotransposition events caused by the insertion of human LINE-1, HERVK-10 or mouse VL30 retroelements by spermatozoa in the zygote and their excessive uncontrolled expression at the cleavage stage and further on to the morula and the blastocyst stage.

In conclusion, spermatozoa intracellular milieu probably favors retrotransposition events of endogenous or exogenous origin. The controlled retroelement RNA expression may be beneficial for reproduction. By contrast, the uncontrolled expression and the potential genome remodeling due to exogenous retrotransposon incorporation or endogenous activation could lead to defective genetic constitution and function of spermatozoa as well as to morphological and genetic abnormalities of preimplantation embryos.

\section{Declaration of interest}

The authors declare that there is no conflict of interest that could be perceived as prejudicing the impartiality of the research reported.

\section{Funding}

This research has been co-financed by the European Union (European Social Fund - ESF) and Greek national funds through the Operational Program 'Education and Lifelong Learning' of the National Strategic Reference Framework (NSRF) - Research Funding Program: Heracleitus II, investing in knowledge society through the European Social Fund.

\section{Acknowledgements}

We would like to thank Professor Haig Kazazian for using his LINE constructs.

\section{References}

Becker KG, Swergold GD, Ozato K \& Thayer RE 1993 Binding of the ubiquitous nuclear transcription factor $\mathrm{YY} 1$ to a cis regulatory sequence in the human LINE-1 transposable element. Human Molecular Genetics 2 1697-1702. (doi:10.1093/hmg/2.10.1697)

Dimitriadou E, Noutsopoulos D, Markopoulos G, Vlaikou AM, Mantziou S, Traeger-Synodinos J, Kanavakis E, Chrousos GP, Tzavaras T \& Syrrou M 2013 Abnormal DLK1/MEG3 imprinting correlates with decreased HERV-K methylation after assisted reproduction and preimplantation genetic diagnosis. Stress 16 689-697. (doi:10.3109/10253890.2013.817554)

Ergun S, Buschmann C, Heukeshoven J, Dammann K, Schnieders F, Lauke H, Chalajour F, Kilic N, Stratling WH \& Schumann GG 2004 Cell type-specific expression of LINE-1 open reading frames 1 and 2 in fetal and adult human tissues. Journal of Biological Chemistry 279 27753-27763. (doi:10.1074/jbc.M312985200)

Francolini M, Lavitrano M, Lamia CL, French D, Frati L, Cotelli F \& Spadafora C 1993 Evidence for nuclear internalization of exogenous DNA into mammalian sperm cells. Molecular Reproduction and Development 34 133-139. (doi:10.1002/(ISSN)1098-2795)

Georgiou I, Noutsopoulos D, Dimitriadou E, Markopoulos G, Apergi A, Lazaros L, Vaxevanoglou T, Pantos K, Syrrou M \& Tzavaras T 2009 Retrotransposon RNA expression and evidence for retrotransposition events in human oocytes. Human Molecular Genetics 18 1221-1228. (doi:10.1093/hmg/ddp022)

Giordano R, Magnano AR, Zaccagnini G, Pittoggi C, Moscufo N, Lorenzini R \& Spadafora C 2000 Reverse transcriptase activity in mature spermatozoa of mouse. Journal of Cell Biology 148 1107-1114. (doi:10.1083/jcb.148.6.1107)

Goodier JL \& Kazazian HH Jr 2008 Retrotransposons revisited: the restraint and rehabilitation of parasites. Cell 135 23-35. (doi:10.1016/j. cell.2008.09.022)

Grunewald S, Paasch U, Glander HJ \& Anderegg U 2005 Mature human spermatozoa do not transcribe novel RNA. Andrologia 37 69-71. (doi:10.1111/and.2005.37.issue-2-3) 
Gur Y \& Breitbart H 2006 Mammalian sperm translate nuclear-encoded proteins by mitochondrial-type ribosomes. Genes \& Development 20 411-416. (doi:10.1101/gad.367606)

Kano H, Godoy I, Courtney C, Vetter MR, Gerton GL, Ostertag EM \& Kazazian HH Jr 2009 L1 retrotransposition occurs mainly in embryogenesis and creates somatic mosaicism. Genes \& Development 23 1303-1312. (doi:10.1101/gad.1803909)

Kazazian HH Jr 2004 Mobile elements: drivers of genome evolution. Science 303 1626-1632. (doi:10.1126/science.1089670)

Krawetz SA, Kruger A, Lalancette C, Tagett R, Anton E, Draghici S \& Diamond MP 2011 A survey of small RNAs in human sperm. Human Reproduction 26 3401-3412. (doi:10.1093/humrep/der329)

Kurose K, Hata K, Hattori M \& Sakaki Y 1995 RNA polymerase III dependence of the human L1 promoter and possible participation of the RNA polymerase II factor YY1 in the RNA polymerase III transcription system. Nucleic Acids Research 23 3704-3709. (doi:10.1093/nar/23.18.3704)

Lander ES, Linton LM, Birren B, Nusbaum C, Zody MC, Baldwin J, Devon K, Dewar K, Doyle M, FitzHugh W et al. 2001 Initial sequencing and analysis of the human genome. Nature 409 860-921. (doi:10.1038/35057062)

Maione B, Pittoggi C, Achene L, Lorenzini R \& Spadafora C 1997 Activation of endogenous nucleases in mature sperm cells upon interaction with exogenous DNA. DNA and Cell Biology 16 1087-1097. (doi:10.1089/ dna.1997.16.1087)

Miller D 2000 Analysis and significance of messenger RNA in human ejaculated spermatozoa. Molecular Reproduction and Development 56 259-264. (doi:10.1002/(ISSN)1098-2795)

Moran JV, Holmes SE, Naas TP, DeBerardinis RJ, Boeke JD \& Kazazian HH Jr 1996 High frequency retrotransposition in cultured mammalian cells. Cell 87 917-927. (doi:10.1016/S0092-8674(00)81998-4)

Muotri AR, Chu VT, Marchetto MC, Deng W, Moran JV \& Gage FH 2005 Somatic mosaicism in neuronal precursor cells mediated by L1 retrotransposition. Nature 435 903-910. (doi:10.1038/ nature03663)

Noutsopoulos D, Vartholomatos G, Kolaitis N \& Tzavaras T 2006 SV40 large T antigen up-regulates the retrotransposition frequency of viral-like 30 elements. Journal of Molecular Biology 361 450-461. (doi:10.1016/ j.jmb.2006.06.030)

Ostertag EM \& Kazazian HH Jr 2001 Biology of mammalian L1 retrotransposons. Annu Rev Genet 35 501-538. (doi:10.1146/annurev. genet.35.102401.091032)

Ostertag EM, Prak ET, DeBerardinis RJ, Moran JV \& Kazazian HH Jr 2000 Determination of L1 retrotransposition kinetics in cultured cells. Nucleic Acids Research 28 1418-1423. (doi:10.1093/ nar/28.6.1418)

Pittoggi C, Zaccagnini G, Giordano R, Magnano AR, Baccetti B, Lorenzini R \& Spadafora C 2000 Nucleosomal domains of mouse spermatozoa chromatin as potential sites for retroposition and foreign DNA integration. Molecular Reproduction and Development $\mathbf{5 6}$ 248-251. (doi:10.1002/(ISSN)1098-2795)

Pittoggi C, Beraldi R, Sciamanna I, Barberi L, Giordano R, Magnano AR, Torosantucci L, Pescarmona E \& Spadafora C 2006 Generation of biologically active retro-genes upon interaction of mouse spermatozoa with exogenous DNA. Molecular Reproduction and Development 73 1239-1246. (doi:10.1002/(ISSN)1098-2795)

Premkumar E \& Bhargava PM 1972 Transcription and translation in bovine spermatozoa. Nature: New Biology 240 139-143. (doi:10.1038/ newbio240139a0)

Rosser JM \& An W 2012 L1 expression and regulation in humans and rodents. Frontiers in Bioscience 4 2203-2225. (doi:10.2741/e537)

Tzavaras T, Kalogera C, Eftaxia S, Saragosti S \& Pagoulatos GN 1998 Clone-specific high-frequency retrotransposition of a recombinant virus containing a VL30 promoter in SV40-transformed NIH3T3 cells. Biochimica et Biophysica Acta 1442 186-198. (doi:10.1016/S01674781(98)00164-X)

van den Hurk JA, Meij IC, Seleme MC, Kano H, Nikopoulos K, Hoefsloot LH, Sistermans EA, de Wijs IJ, Mukhopadhyay A, Plomp AS et al. 2007 L1 retrotransposition can occur early in human embryonic development. Human Molecular Genetics 16 1587-1592. (doi:10.1093/ hmg/ddm108)

Watanabe T, Takeda A, Tsukiyama T, Mise K, Okuno T, Sasaki H, Minami N \& Imai H 2006 Identification and characterization of two novel classes of small RNAs in the mouse germline: retrotransposon-derived siRNAs in oocytes and germline small RNAs in testes. Genes \& Development 20 1732-1743. (doi:10.1101/gad.1425706)

Waterston RH, Lindblad-Toh K, Birney E, Rogers J, Abril JF, Agarwal P, Agarwala R, Ainscough R, Alexandersson M, An P et al. 2002 Initial sequencing and comparative analysis of the mouse genome. Nature $\mathbf{4 2 0}$ 520-562. (doi:10.1038/nature01262)

Yu F, Zingler N, Schumann G \& Stratling WH 2001 Methyl-CpG-binding protein 2 represses LINE-1 expression and retrotransposition but not Alu transcription. Nucleic Acids Research 29 4493-4501. (doi:10.1093/ nar/29.21.4493)

Zoraqi G \& Spadafora C 1997 Integration of foreign DNA sequences into mouse sperm genome. DNA and Cell Biology 16 291-300. (doi:10.1089/ dna.1997.16.291)

Received 21 September 2015

First decision 19 October 2015

Revised manuscript received 5 May 2016

Accepted 7 June 2016 